\title{
Network Disruption and Cerebrospinal Fluid Amyloid-Beta and Phospho-Tau Levels in Mild Cognitive Impairment
}

\author{
Leonides Canuet, ${ }^{1,2 \star}$ Sandra Pusil, ${ }^{1 \star}$ María Eugenia López, ${ }^{1,2}$ Ricardo Bajo, ${ }^{1,2}$ José Ángel Pineda-Pardo, ${ }^{1,2,3}$ Pablo Cuesta, ${ }^{1,4}$ \\ Gerardo Gálvez, ${ }^{1,5}$ José María Gaztelu, ${ }^{6}$ Daniel Lourido, ${ }^{7}$ Guillermo García-Ribas, ${ }^{7}$ and Fernando Maestú ${ }^{1,2,4}$ \\ ${ }^{1}$ Laboratory of Cognitive and Computational Neuroscience, Center for Biomedical Technology, Madrid Polytechnic University and Complutense University \\ of Madrid, 28223 Madrid, Spain, ${ }^{2}$ Networking Research Center on Bioengineering, Biomaterials and Nanomedicine (CIBER-BBN), ${ }^{3}$ CINAC, HM Puerta del \\ Sur, Hospitales de Madrid, Móstoles, 28938 Madrid, Spain, ${ }^{4}$ Department of Basic Psychology II, School of Psychology, Complutense University of Madrid, \\ 28040 Madrid, Spain, 5 Instrumentation and Applied Acoustic Research Group, 28031 Madrid, Spain, ${ }^{6}$ Department of Experimental Neurology, Ramon y \\ Cajal University Hospital, Instituto Ramón y Cajal de Investigación Sanitaria, 28034 Madrid, Spain, and Department of Neurology, Ramon y Cajal \\ University Hospital, 28034 Madrid, Spain
}

Synaptic dysfunction is a core deficit in Alzheimer's disease, preceding hallmark pathological abnormalities. Resting-state magnetoencephalography (MEG) was used to assess whether functional connectivity patterns, as an index of synaptic dysfunction, are associated with CSF biomarkers [i.e., phospho-tau (p-tau) and amyloid beta (A $\beta 42)$ levels]. We studied 12 human subjects diagnosed with mild cognitive impairment due to Alzheimer's disease, comparing those with normal and abnormal CSF levels of the biomarkers. We also evaluated the association between aberrant functional connections and structural connectivity abnormalities, measured with diffusion tensor imaging, as well as the convergent impact of cognitive deficits and CSF variables on network disorganization. One-third of the patients converted to Alzheimer's disease during a follow-up period of 2.5 years. Patients with abnomal CSF p-tau and A $\beta 42$ levels exhibited both reduced and increased functional connectivity affecting limbic structures such as the anterior/posterior cingulate cortex, orbitofrontal cortex, and medial temporal areas in different frequency bands. A reduction in posterior cingulate functional connectivity mediated by p-tau was associated with impaired axonal integrity of the hippocampal cingulum. We noted that several connectivity abnormalities were predicted by CSF biomarkers and cognitive scores. These preliminary results indicate that CSF markers of amyloid deposition and neuronal injury in early Alzheimer's disease associate with a dual pattern of cortical network disruption, affecting key regions of the default mode network and the temporal cortex. MEG is useful to detect early synaptic dysfunction associated with Alzheimer's disease brain pathology in terms of functional network organization.

Key words: Alzheimer's disease; brain network; functional connectivity; magnetoencephalography; mild cognitive impairment; structural connectivity

Significance Statement

In this preliminary study, we used magnetoencephalography and an integrative approach to explore the impact of CSF biomarkers, neuropsychological scores, and white matter structural abnormalities on neural function in mild cognitive impairment. Disruption in functional connectivity between several pairs of cortical regions associated with abnormal levels of biomarkers, cognitive deficits, or with impaired axonal integrity of hippocampal tracts. Amyloid deposition and tau protein-related neuronal injury in early Alzheimer's disease are associated with synaptic dysfunction and a dual pattern of cortical network disorganization (i.e., desynchronization and hypersynchronization) that affects key regions of the default mode network and temporal areas.

\section{Introduction}

"Mild cognitive impairment (MCI) due to Alzheimer's disease" refers to the symptomatic predementia phase of Alzheimer's dis-

Received Feb. 20, 2015; revised May 25, 2015; accepted June 11, 2015.

Author contributions: L.C., S.P., R.B., G.G.-R., and F.M. designed research; L.C., S.P., M.E.L., J.M.G., D.L., G.G.-R., and F.M. performed research; J.A.P.-P. and P.C. contributed unpublished reagents/analytic tools; L.C., S.P., M.E.L., R.B., J.A.P.-P., and G.G. analyzed data; L.C., S.P., M.E.L., R.B., J.A.P.-P., and F.M. wrote the paper. ease. The likelihood that MCI is due to Alzheimer's disease is strongly influenced by biomarkers. Accordingly, patients with MCI who progress to Alzheimer-type dementia will likely show positive biomarkers of both amyloid-beta $(\mathrm{A} \beta)$ deposition and 
tau-related neuronal injury (Albert et al., 2011). Low levels of CSF $\mathrm{A} \beta 42$ peptides indicate increased $\mathrm{A} \beta$ deposition in the brain and high levels of CSF tau protein derived from damaged neuronal microtubules are considered reliable biological markers of Alzheimer's disease and predictors of MCI converters (Vlassenko et al., 2012). In particular, the phosphorylated form of tau or "phospho-tau" (p-tau) appears to provide a high specificity in the diagnosis of Alzheimer's disease compared with total tau (Andreasen et al., 2003).

Increasing evidence indicates that synaptic dysfunction is a core deficit in Alzheimer's disease, preceding hallmark pathological abnormalities (Kim et al., 2013; Grandjean et al., 2014). Soluble $\mathrm{A} \beta$ oligomers and tau fibrillar lesions affect synaptic plasticity and cause synaptic loss, leading to impairment of neural networks involved in memory and cognition. For this reason, MCI and Alzheimer's disease are best characterized as a disruption of functional and structural integration of neural systems rather than as regionally localized abnormalities (Garcés et al., 2014; Klupp et al., 2014; López et al., 2014; Pineda-Pardo et al., 2014). Magnetoencephalography (MEG) is a neurophysiological technique able to measure neural activity (i.e., postsynaptic potentials) directly and noninvasively with a high temporal resolution (Stam, 2010; Canuet et al., 2012). Therefore, MEG is suitable for measuring brain dynamics and synaptic dysfunction in cognitive disorders using functional connectivity.

To date, little is known about whether the levels of p-tau and $\mathrm{A} \beta 42$ in the CSF are associated with the disruption of brain functional networks in patients with MCI. In this study, we used MEG to determine biomagnetic patterns of resting-state functional connectivity associated with abnomal CSF levels of p-tau and $\mathrm{A} \beta 42$ in patients with MCI. We also aimed to determine an association between these patterns and structural connectivity abnormalities and the convergent impact of neuropsychological scores and CSF variables on network disorganization. We hypothesize that the CSF levels of both p-tau and A $\beta 42$ would cause synaptic disruption and thus impair the organization of anatomofunctional brain networks that leads to cognitive impairment.

\section{Materials and Methods}

MEG recordings were obtained from 12 patients with MCI (mean age: $69.42 \pm 8.19$; female: $n=10$ ), recruited from the Ramón y Cajal Hospital in Madrid, Spain. All of them were right-handed. MCI diagnosis was made according to the National Institute on Aging-Alzheimer Association (NIA-AA) clinical criteria (Albert et al., 2011). In addition to clinical features, evidence of amyloid deposition and/or neuronal injury as indicated by CSF biomarkers was used to diagnose these patients with MCI due to Alzheimer's disease as intermediate or high likelihood and MCI converters. With regard to neuronal injury biomarkers, we focused on CSF p-tau protein because it is considered to be more specific to Alzheimer's-type dementia compared with CSF total tau (Andreasen et al., 2003).

p-tau and $A \beta 42$ levels in the CSF were measured using ELISA following supplier recommendations (Innotest; Innogenetics). Cutoff points were obtained by our laboratory and sent to the Alzheimer's Association Quality Control Program Work Group blindly to clinical or neurophysiological data (Mattsson et al., 2013). For each CSF biomarker, patients were divided into CSF-abnormal (biomarker positive) and CSF-normal (the biomarker-negative) group. Those with CSF levels of $\mathrm{p}$-tau $>60$ $\mathrm{pg} / \mathrm{ml}$ and $\mathrm{A} \beta 42<485 \mathrm{pg} / \mathrm{ml}$ made up the CSF-abnormal group for the

\section{*L.C. and S.P. contributed equally to this work.}

Correspondence should be addressed to L. Canuet, Center for Biomedical Technology, Campus Montegancedo, M40, km 38, 28223 Pozuelo de Alarcon, Madrid, Spain. E-mail: leocanon2002@gmail.com.

DOI:10.1523/JNEUROSCI.0704-15.2015

Copyright $\odot 2015$ the authors $\quad 0270-6474 / 15 / 3510326-06 \$ 15.00 / 0$ corresponding biomarker. Otherwise, they were included in the CSFnormal group. Neuropsychological assessment included the Clinical Dementia Rate (CDR), the Mini Mental State Examination (MMSE), and the immediate and free recall tests of the 7 Minute Screening Neurocognitive Battery (7MS).

MEG recordings were acquired with a 306-channel Vectorview system (Elekta-Neuromag). Patients were in an awake, resting state with their eyes closed. For each subject, 5 min task-free data were recorded. The sampling frequency applied was $1000 \mathrm{~Hz}$. Recordings were filtered offline and corrected for head movements with a temporal signal space separation with movement compensation (tsss-mc) method (Maxfilter 2.2 software). Analyses were performed for alpha $(8-12 \mathrm{~Hz})-$, beta (12-30 $\mathrm{Hz})$-, and gamma ( $30-45 \mathrm{~Hz})$-frequency bands based on evidence for a role of alpha-band connectivity in the diagnosis of MCI (Garcés et al., 2014; López et al., 2014) and the significance of alpha-, beta-, and gamma-frequency bands to resting-state global network function (Hipp et al., 2012).

The scalp and cortex surface were extracted from each subject's MRI volumes and imported into Brainstorm (http://neuroimage.usc.edu/ brainstorm), the open-source software used for source reconstruction and connectivity analysis (Tadel et al., 2011). The cortex surface was divided into 88 regions of interest (ROIs) based on the Brainstorm default atlas. The source activity for each subject and frequency band was computed using the weighted minimum norm estimation with the default settings on Brainstorm. For the cortex, the source activity obtained was projected onto the default anatomy of Brainstorm. After source map projection onto the default anatomy, the time series of cortical ROIs were extracted.

The phase-locking value (PLV) algorithm was used to measure functional connectivity between all pairs of regions $(88 \times 88)$ for each frequency band (Lachaux et al., 1999). Phase synchronization measures are based on the hypothesis that the difference of phases between two phaselocked systems must be nonuniform, so the degree of nonuniformity must be a good estimator of the coupling level. For analysis, first, data were band-pass filtered using a zero-phase distortion finite impulse response filter with a bandwidth of $2 \mathrm{~Hz}$. Then, the complex analytic signal from each of the brain regions was obtained by using the Hilbert transform (for details, see Pereda et al., 2005). Subsequently, the PLV between the time series $x(k)$ and $y(k)$ from two brain regions is defined as follows:

$$
P L V_{x y}=\frac{1}{N} \sum_{k=1}^{N}\left|e^{i\left(\phi_{x}(\mathrm{k})-\phi_{x}(\mathrm{k})\right)}\right|
$$

where $N$ is the length of the time series, and $\emptyset_{x}(k)\left(\right.$ resp. $\left.\emptyset_{y}(k)\right)$ is the phase of $x(k)$ (resp. $y(k)$ ). Finally, the PLVs were averaged for each frequency band.

Hippocampal volumes were measured as anatomical evidence of the degree of brain atrophy that characterizes MCI (Albert et al., 2011). A high-resolution T1-weighted MRI was acquired for each subject with a Philips 1.5 tesla MRI scanner using a fast-field echo sequence. Freesurfer software (version 5.1.0) and its specialized tool for automated subcortical segmentation were used to segment the subject's T1-weighted volume into different regions. Finally, hippocampal volume was normalized with the overall intracranial volume to account for differences in head volume over subjects.

Diffusion tensor images (DTIs) were obtained using a linear leastsquares approach in FSL-FDT and several scalar images representing the shape of the diffusion tensor were obtained: fractional anisotropy, mean diffusivity, radial diffusivity, and axial diffusivity. Images with $b=0$ $\mathrm{s} / \mathrm{mm}^{2}$ (i.e., $b_{0}$ images) were realigned to brain extracted T1-weighted images using an affine linear transformation in FSL-FLIRT, with mutual information as cost function and nearest neighbor interpolation. Linear affine transformations followed by nonlinear local deformations using FSL-FNIRT were applied to normalize brain-extracted T1-weighted images into the MNI brain template of $1 \mathrm{~mm}^{3}$ isotropic. Transformations were concatenated and applied inversely to transform the JHU white matter tractography atlas (Hua et al., 2008) into the diffusion subjectspecific space. The tracts of interest included the anterior thalamic radiation, corticospinal tract, cingulum, hippocampal cingulum, forceps 
Table 1. Patient characteristics

\begin{tabular}{|c|c|c|c|c|c|c|}
\hline & \multicolumn{3}{|l|}{ p-tau } & \multicolumn{3}{|l|}{$A \beta(1-42)$} \\
\hline & Abnormal $(n=7)$ & Normal $(n=5)$ & $p$-value & Abnormal $(n=8)$ & Normal $(n=4)$ & $p$-value \\
\hline Age & $69.86 \pm 6.51$ & $68.80 \pm 10.96$ & 0.85 & $66.50 \pm 7.03$ & $75.25 \pm 7.93$ & 0.12 \\
\hline MMSE score & $26 \pm 1.09$ & $27.80 \pm 2.49$ & 0.19 & $27.57 \pm 1.99$ & $25.50 \pm 1.29$ & 0.07 \\
\hline Free recall & $12.17 \pm 5.67$ & $14.20 \pm 2.19$ & 0.45 & $12.71 \pm 5.28$ & $13.75 \pm 2.63$ & 0.67 \\
\hline Hippocampal volume left & $0.0021 \pm 0.00042$ & $0.0024 \pm 0.00032$ & 0.11 & $0.0024 \pm 0.00045$ & $0.0021 \pm 0.00033$ & 0.32 \\
\hline Hippocampal volume right & $0.002 \pm 0.00041$ & $0.0024 \pm 0.00027$ & 0.11 & $0.0022 \pm 0.00041$ & $0.0019 \pm 0.00027$ & 0.18 \\
\hline
\end{tabular}

Data are mean \pm SD unless otherwise noted.

major and minor, inferior fronto-occipital fasciculus, superior longitudinal fasciculus, inferior longitudinal fasciculus, and uncinate fasciculus.

For statistical analyses, based on the non-Gaussian distribution of the MEG data, connectivity results were compared between the CSFabnormal and CSF-normal group for each biomarker using the Mann -Whitney $U$ test. A total of 5000 permutations were used to correct for multiple comparisons. The neuropsychological scores were correlated with the values (PLVs) of the functional connections found significantly different between groups and with the CSF levels of each biomarker using Spearman correlation coefficient (uncorrected). For an integrative approach of the impact of neuropsychological scores and CSF variables on neural function, multivariate regression models were built, with these variables acting as predictors and the functional connections as the dependent variables. For DTI analysis, average values within the 20 atlas tracts for the scalar images were obtained and statistical group comparisons were performed using an unpaired $t$ test. The correlation between PLVs and the fractional anisotropy values of the significantly different tracts was measured using Spearman correlation coefficient (uncorrected).

\section{Results}

Demographic and clinical characteristics of the patients are shown in Table 1 . There were no differences in age, cognitive scores, or hippocampal volumes across groups. Abnormal CSF p-tau and A $\beta 42$ levels were found in seven and eight patients, respectively. Five of them were positive for both biomarkers at the time of the study. Four MCI patients converted to Alzheimer's disease during the follow-up period of 2.5 years. These patients showed low levels of $A \beta 42$ and high levels of $\mathrm{p}$-tau in the CSF.

\section{p-tau}

Patients with abnormal CSF p-tau levels showed an abnormal pattern characterized by decreased functional connectivity between the right posterior cingulate cortex and paracentral lobule in alpha band, the right orbitofrontal cortex and contralateral calcarine area in the beta band, and the right paracentral lobule and lingual cortex in the gamma band. Increased connectivity was observed between the right supplementary motor area and the contralateral cuneus in the alpha band. In addition, there was increased intrahemispheric connectivity between the right anterior cingulate cortex and medial temporal area in the beta band and between the right middle frontal cortex and anterior temporal area in the gamma band (Fig. 1, Table 2).

\section{A $\beta 42$}

Patients with abnormal CSF A $\beta 42$ levels exhibited decreased functional connectivity between right temporal areas and several cortical regions of the frontal, parietal, and temporal lobes compared with patients with normal CSF levels. Decreased alpha connectivity was found between the right posterior cingulate cortex and the ipsilateral middle temporal cortex, as well as between the right medial temporal and left superior parietal cortex. In the beta band, there was decreased connectivity between the right inferior temporal cortex and the ipsilateral precentral area. These areas also exhibited decreased connectivity in the gamma band. In addition, a decrease in gamma connectivity was found between the right superior temporal cortex and the contralateral anterior temporal area. Abnormal CSF A $\beta 42$ levels were also linked to a hyperconnectivity pattern. There was an increase in functional connectivity of the right medial-superior frontal cortex with the right fusiform area in the beta band (Fig. 2, Table 2).

\section{Cognitive scores}

An increase in beta connectivity between the right anterior cingulate cortex and the ipsilateral medial temporal cortex showed a negative correlation with the MMSE score $(r=-0.79 ; p=0.003)$ and the free recall score $(r=-0.67 ; p=0.023)$ in patients with abnormal CSF p-tau levels relative to those with normal levels. In addition, a decrease in interhemispheric connectivity between the right posterior cingulate cortex and paracentral area correlated positively with the immediate recall score $(r=0.72 ; p=$ 0.012 ). In the CSF-abnormal $A \beta 42$ group, a positive correlation was found between the MMSE score and decreased beta connectivity between the inferior temporal and precentral cortex $(r=$ $0.77 ; p=0.005)$. A correlation analysis of cognitive scores and CSF biomarkers revealed that only the MMSE score correlated with the CSF levels of both p-tau $(r=-0.68 ; p=0.021)$ and $\mathrm{A} \beta 42(r=0.82 ; p=0.002)$. To explore the convergent impact of cognitive scores and CSF values on brain functional connectivity, we performed multivariate regression analysis with the significant connections as the dependent variables (Table 2). We found that the CSF levels of the biomarkers predicted most of the aberrant functional connections seen in these MCI patients. In addition, the free recall score showed a predictive value for intrahemispheric anterior cingulate-medial temporal connectivity, whereas the MMSE score predicted functional connectivity between the inferior temporal and precentral cortex.

\section{Correlation between functional connectivity and structural connectivity}

The correlation analysis of the significant functional connections and structural connectivity measures revealed that the decrease in alpha synchronization between the right posterior cingulate and left paracentral lobule mediated by p-tau correlated positively with the fractional anisotropy value of the right hippocampal cingulum $(r=0.81 ; p=0.003)$. This fractional anisotropy reduction correlated with CSF p-tau levels $(r=-0.70 ; p=0.016)$. There was no significant correlation between fractional anisotropy and cognitive scores.

\section{Discussion}

In this study, we assessed whether functional connectivity patterns were associated with CSF biomarkers (i.e., p-tau, and A $\beta 42$ concentrations) in patients with MCI due to Alzheimer's disease. 

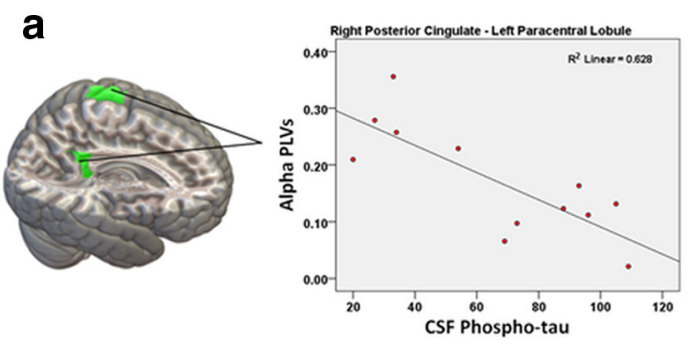

b
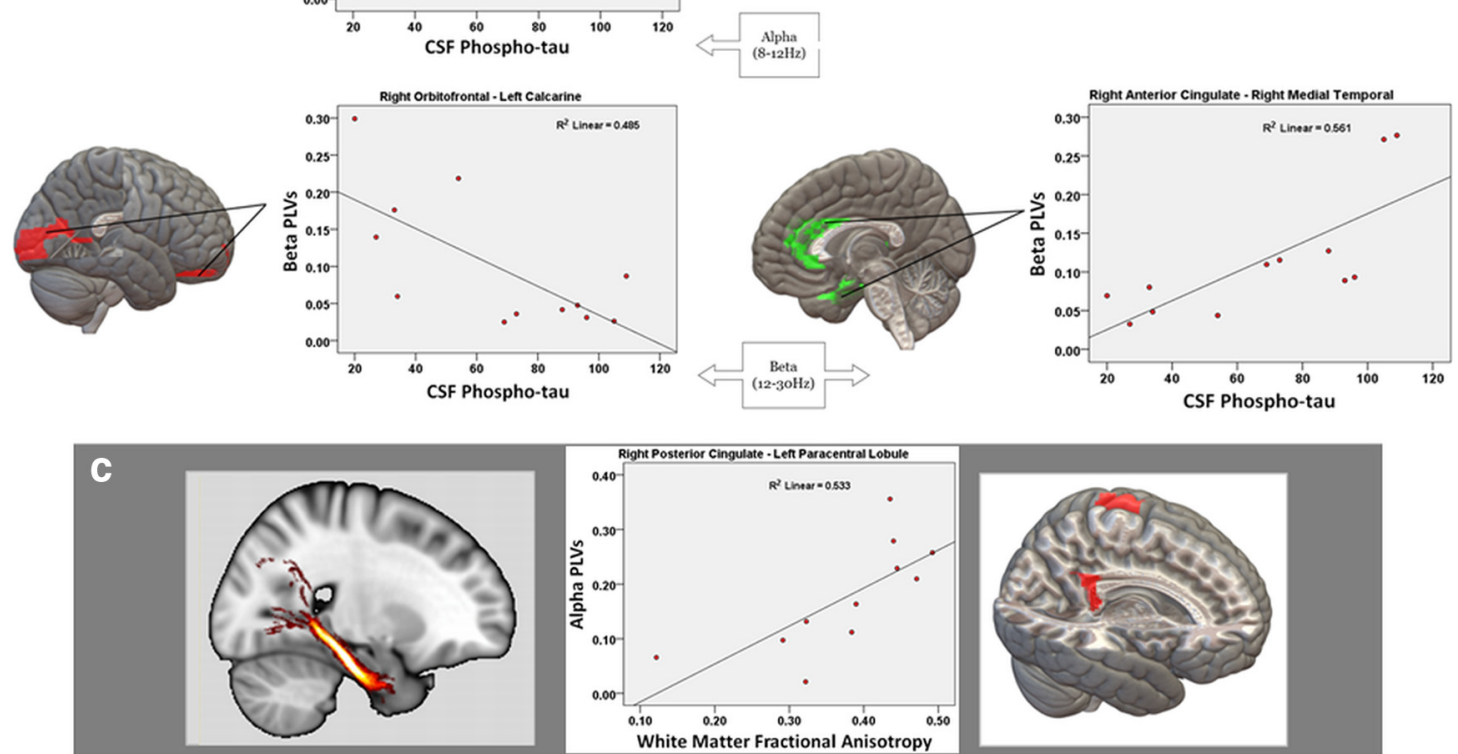

Figure 1. Pairs of regions showing significant functional connectivity abnormalities predicted by p-tau levels in the CSF. Cortical regions show p-tau-related desynchronization (a) and $p$-tau-related hypersynchronization $(\boldsymbol{b})$. Scatterplots of the correlations between these functional connections and p-tau levels are provided. $\boldsymbol{c}$, Significant correlation between functional and structural connections. DTls of the right hippocampal cingulum and the desynchronized regions show a significant correlation with fractional anisotropy. Scatterplots of the correlation between fractional anisotropy and connectivity values are provided.

Table 2. Phase synchronization and correlation values of cortical regions showing significantly different functional connectivity across biomarker groups

\begin{tabular}{|c|c|c|c|c|c|c|c|c|c|}
\hline \multirow[b]{2}{*}{ Frequency bands } & \multirow[b]{2}{*}{ Pairs of regions } & \multicolumn{2}{|c|}{$\mathrm{MCl}$ groups mean connectivity } & \multicolumn{2}{|c|}{$\begin{array}{l}\text { Connectivity } \\
\text { (statistics) }\end{array}$} & \multicolumn{4}{|c|}{ Multivariate regression } \\
\hline & & p-tau abnormal & p-tau normal & $\chi^{2}$ & $p$-value & Model's adj. $R^{2}$ & Predictor & $F$ & $p$-value \\
\hline \multirow[t]{2}{*}{ Alpha $(8-12 \mathrm{~Hz})$} & Posterior cingulate (R) and paracentral lobule (L) & 0.10 & 0.21 & 5.55 & 0.018 & 0.64 & CSF p-tau & 9.42 & 0.028 \\
\hline & Cuneus $(\mathrm{L})$ and supplementary motor cortex (R) & 0.12 & 0.06 & 8.06 & 0.004 & 0.28 & - & - & - \\
\hline \multirow{3}{*}{ Beta $(12-30 \mathrm{~Hz})$} & Orbitofrontal cortex (R) and calcarine (L) & 0.04 & 0.18 & 7.18 & 0.007 & 0.79 & CSF p-tau & 25.97 & 0.004 \\
\hline & Anterior cingulate $(\mathrm{R})$ and medial temporal cortex $(\mathrm{R})$ & 0.15 & 0.05 & 8.08 & 0.004 & 0.76 & Free recall & 9.96 & 0.025 \\
\hline & & & & & & & CSF p-tau & 9.21 & 0.029 \\
\hline \multirow[t]{3}{*}{ Gamma $(30-45 \mathrm{~Hz})$} & Paracentral lobule (R) and lingual (L) & 0.05 & 0.14 & 7.10 & 0.008 & 0.53 & - & - & - \\
\hline & Middle frontal cortex (R) and anterior temporal (R) & 0.14 & 0.04 & 8.08 & 0.004 & 0.25 & - & - & - \\
\hline & & $A \beta 42$ abnormal & $A \beta 42$ normal & & & & & & \\
\hline \multirow[t]{2}{*}{ Alpha $(8-12 \mathrm{~Hz})$} & Posterior cingulate (R) and middle temporal cortex (R) & 0.13 & 0.32 & 7.31 & 0.006 & 0.14 & - & - & - \\
\hline & Medial temporal cortex (R) and superior parietal cortex (L) & 0.18 & 0.30 & 6.49 & 0.010 & 0.64 & CSF A $\beta 42$ & 8.71 & 0.032 \\
\hline \multirow[t]{3}{*}{ Beta $(12-30 \mathrm{~Hz})$} & Inferior temporal cortex (R) and precentral (R) & 0.04 & 0.16 & 7.38 & 0.006 & 0.85 & MMSE & 7.24 & 0.040 \\
\hline & & & & & & & CSF A $\beta 42$ & 41.22 & 0.001 \\
\hline & Medial prefrontal cortex (R) and fusiform (R) & 0.13 & 0.06 & 6.40 & 0.011 & 0.68 & CSF A $\beta 42$ & 14.76 & 0.012 \\
\hline \multirow[t]{2}{*}{ Gamma $(30-45 \mathrm{~Hz})$} & Inferior temporal cortex $(\mathrm{R})$ and precentral $(\mathrm{R})$ & 0.08 & 0.18 & 7.30 & 0.006 & 0.84 & CSF A $\beta 42$ & 21.45 & 0.006 \\
\hline & Superior temporal $(\mathrm{R})$ and anterior temporal $(\mathrm{L})$ & 0.05 & 0.13 & 6.41 & 0.011 & 0.67 & CSF A $\beta 42$ & 22.30 & 0.005 \\
\hline
\end{tabular}

In addition, we evaluated the relationship of the aberrant functional connections with structural connectivity abnormalities and cognitive deficits. Patients with p-tau pathology had reduced functional connectivity affecting limbic structures such as the posterior cingulate and the orbitofrontal cortex, as well as medial parietal areas, in particular the paracentral lobule (Fig. 1). CSF p-tau levels predicted abnormalities specifically in the alpha- and beta-frequency range (Fig. 1, Table 2). It is well established that tau hyperphosphorylation is associated with intracellular aggregation of neurofibrillary tangles and Alzheimer's-disease-related neurodegeneration (Vlassenko et al., 2012; van Rossum et al.,
2012). Recent evidence suggests that, even in the preclinical stage of Alzheimer's disease, limbic structures are affected by tau pathology. There is also evidence that limbic dysfunction involves primarily the entorhinal cortex and then spreads to the parietal cortex (Khan et al., 2014) and inferior frontal areas, including the orbitofrontal cortex (Serrano-Pozo et al., 2011). This phenomenon is thought to be due to synaptic loss and trans-synaptic spread of pathological forms of tau through brain circuits involved in learning and memory formation (Spires-Jones and Hyman, 2014), which can explain the typical cognitive deficits seen in MCI due to Alzheimer's disease. 

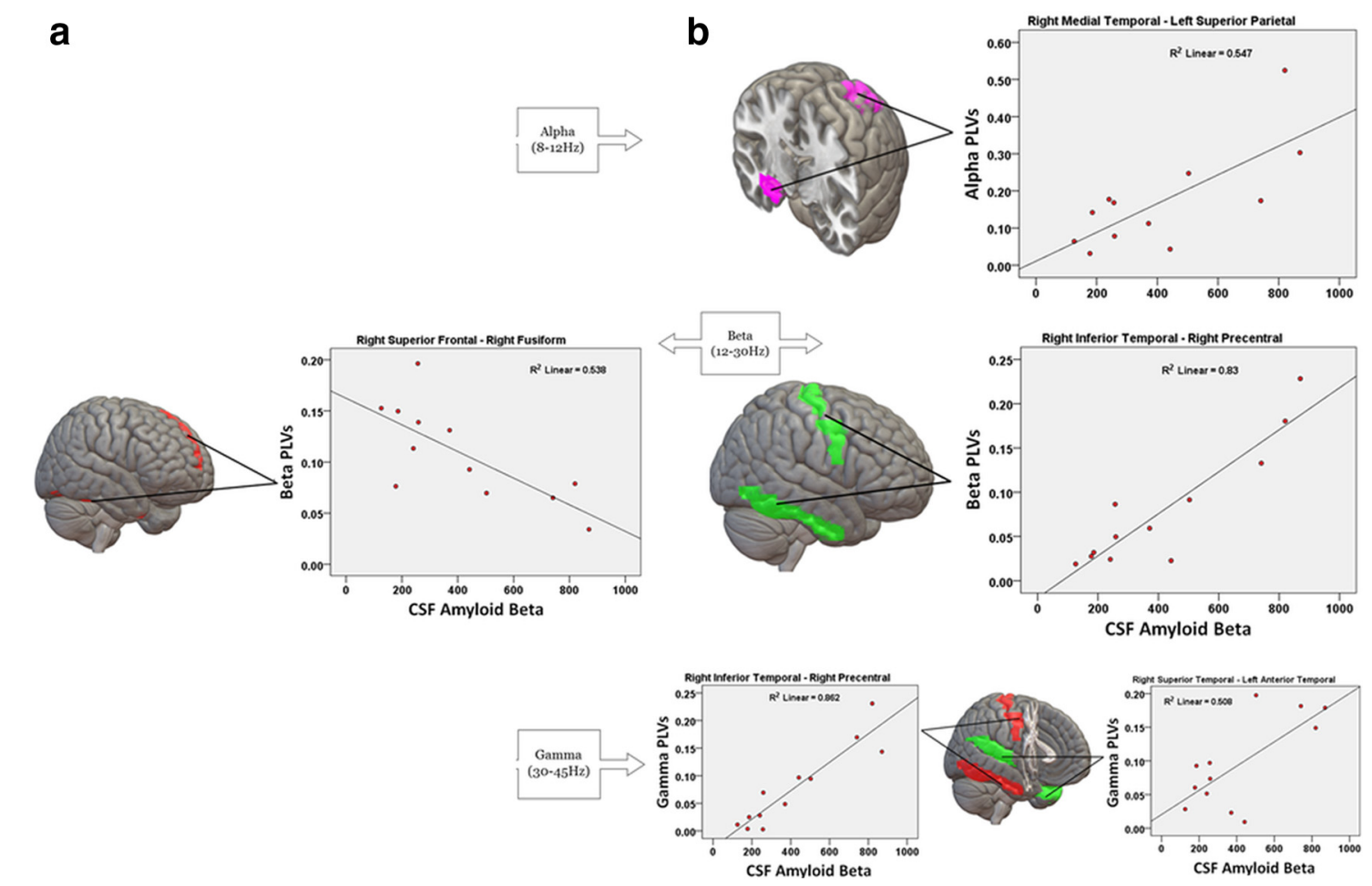

Figure 2. Pairs of regions showing significant functional connectivity abnormalities predicted by A $\beta 42$ levels in the CSF. Cortical regions show A $\beta 42$-related hypersynchronization (a) and $A \beta 42$-related desynchronization (b). Scatterplots of the correlations between these functional connections and $A \beta 42$ levels are provided.

Interestingly, the reduced functional connectivity of the posterior cingulate cortex mediated by p-tau correlated positively with impaired axonal integrity of the ipsilateral hippocampal cingulum, as indicated by a significant decrease in fractional anisotropy (Fig. 1c). This supports the notion that the disruption of anatomical networks influences brain organization at the functional level, resulting in the manifestations of MCI syndrome (Pineda-Pardo et al., 2014). Consistent with this finding, an investigation performed by Amlien et al. (2013), in which CSF biomarkers were also used, revealed regional white matter loss in the right hippocampal cingulum in association with tau pathology. Together, these results and ours suggest a strong relationship among synaptic dysfunction, axonal disintegration in limbic areas, and tau pathology as key pathophysiological mechanisms involved in MCI due to Alzheimer's disease.

In addition to decreased functional connectivity, patients with abnormal CSF p-tau levels also showed a hyperconnectivity pattern affecting mainly the anterior cingulate and medial temporal cortex (Fig. $2 b$ ), which was predicted by the free recall score (Table 2). Therefore, this increase in connectivity affecting the anterior cingulate cortex may reflect a pathological activity that exerts a negative impact on cognition, particularly on memory function, and may increase the risk of conversion to Alzheimer's disease (López et al., 2014).

A reduction in CSF A $\beta 42$ levels was associated with decreased functional connectivity of the posterior cingulate cortex and neocortical temporal regions. The lateral parietal and medial temporal regions were also involved. Disrupted connectivity of the inferior temporal cortex in the beta band was predicted by the MMSE score. The posterior cingulate and lateral parietal cortex are key regions of the default mode network, a brain circuit typically active during rest, the structures of which are vulnerable to A $\beta$ deposition (Sheline et al., 2010; Li et al., 2013). Consistent with our findings, fMRI and PET studies demonstrated that $\mathrm{A} \beta$ plaques disrupt resting-state connectivity within the default mode network (Sheline et al., 2010; Drzezga et al., 2011) and in the lateral temporal cortex, including inferior temporal areas (Carbonell et al., 2014), in preclinical Alzheimer's disease and MCI.

We also noted a decrease in connectivity of the medial temporal cortex associated with the $A \beta 42$ biomarker. This cortical area is thought to have a low $\mathrm{A} \beta$ burden, particularly in early stages of the disease (Serrano-Pozo et al., 2011). However, the spatial distribution of $\mathrm{A} \beta$ deposition and its effect on hippocampus function during the course of dementia is still a matter of debate. Therefore, although a direct toxic effect of $\mathrm{A} \beta$ plaques on medial temporal structures cannot be ruled out completely, this decrease in connectivity could be mediated by connectional diaschisis (Campo et al., 2012). This phenomenon explains that the intimate anatomo-functional relation between regions showing amyloid accumulation and the hippocampus might affect the ability of this area to establish close communication with other brain regions, resulting in connectivity reduction and consequently in a reduced neuronal input from those regions. Consistent with this assumption, a recent report using CSF biomarkers also suggested an association between decreased CSF A $\beta 42$ and decreases in fMRI functional connectivity in the posterior cingulate and medial temporal cortex in MCI and Alzheimer's disease (Wang et al., 2013).

We found that $\mathrm{A} \beta$-related hypersynchronization in this study was characterized by the involvement of prefrontal connections. Because this abnormality showed no association with cognitive deficits and the prefrontal cortex is thought not to be affected early in Alzheimer's-type dementia, this increased connectivity might be linked to a compensatory mechanism. This would be in agreement with evidence indicating that patients with Alzheimer's disease use additional neural resources in prefrontal cortex, presumably those mediating executive functions, to compensate 
for losses attributable to the degenerative process of the disease (Grady et al., 2003).

Overall, our results should be considered as preliminary based on the small sample size of MCI patients. Nevertheless, this study indicates that CSF abnormal levels of p-tau and $A \beta 42$ in early Alzheimer's disease are associated with cortical network disruption involving desynchronization and hypersynchronization of key regions of the default mode network; for example, the anterior and posterior cingulate cortex and specific temporal and frontal areas. This dual pattern could represent two sides of a disrupting network that loses equilibrium, leading to cognitive impairment. Upon confirmation of these findings, MEG may potentially be used as a tool to detect early synaptic dysfunction associated with Alzheimer's disease brain pathology in terms of functional network organization.

\section{References}

Albert MS, DeKosky ST, Dickson D, Dubois B, Feldman HH, Fox NC, Gamst A, Holtzman DM, Jagust WJ, Petersen RC, Snyder PJ, Carrillo MC, Thies B, Phelps CH (2011) The diagnosis of mild cognitive impairment due to Alzheimer's disease: recommendations from the National Institute on Aging-Alzheimer's Association workgroups on diagnostic guidelines for Alzheimer's disease. Alzheimers Dement 7:270-279. CrossRef Medline

Amlien IK, Fjell AM, Walhovd KB, Selnes P, Stenset V, Grambaite R, Bjørnerud A, Due-Tønnessen P, Skinningsrud A, Gjerstad L, Reinvang I, Fladby $\mathrm{T}$ (2013) Mild cognitive impairment: cerebrospinal fluid tau biomarker pathologic levels and longitudinal changes in white matter integrity. Radiology 266:295-303. CrossRef Medline

Andreasen N, Sjögren M, Blennow K (2003) CSF markers for Alzheimer's disease: total tau, phospho-tau and Abeta42. World J Biol Psychiatry 4:147-155. CrossRef Medline

Campo P, Garrido MI, Moran RJ, Maestú F, García-Morales I, Gil-Nagel A, del Pozo F, Dolan RJ, Friston KJ (2012) Remote effects of hippocampal sclerosis on effective connectivity during working memory encoding: a case of connectional diaschisis? Cereb Cortex 22:1225-1236. CrossRef Medline

Canuet L, Tellado I, Couceiro V, Fraile C, Fernandez-Novoa L, Ishii R, Takeda M, Cacabelos R (2012) Resting-state network disruption and APOE genotype in alzheimer's disease: a lagged functional connectivity study. PLoS One 7.

Carbonell F, Charil A, Zijdenbos AP, Evans AC, Bedell BJ; Alzheimer's Disease Neuroimaging Initiative (2014) Hierarchical multivariate covariance analysis of metabolic connectivity. J Cereb Blood Flow Metab 34: 1936-1943. CrossRef Medline

Drzezga A, Becker JA, Van Dijk KR, Sreenivasan A, Talukdar T, Sullivan C, Schultz AP, Sepulcre J, Putcha D, Greve D, Johnson KA, Sperling RA (2011) Neuronal dysfunction and disconnection of cortical hubs in nondemented subjects with elevated amyloid burden. Brain 134:1635-1646. CrossRef Medline

Garcés P, Angel Pineda-Pardo J, Canuet L, Aurtenetxe S, López ME, Marcos A, Yus M, Llanero-Luque M, Del-Pozo F, Sancho M, Maestú F (2014) The Default Mode Network is functionally and structurally disrupted in amnestic mild cognitive impairment - a bimodal MEG-DTI study. Neuroimage Clin 6:214-221. CrossRef Medline

Grady CL, McIntosh AR, Beig S, Keightley ML, Burian H, Black SE (2003) Evidence from functional neuroimaging of a compensatory prefrontal network in Alzheimer's disease. J Neurosci 23:986-993. Medline

Grandjean J, Schroeter A, He P, Tanadini M, Keist R, Krstic D, Konietzko U, Klohs J, Nitsch RM, Rudin M (2014) Early alterations in functional connectivity and white matter structure in a transgenic mouse model of cerebral amyloidosis. J Neurosci 34:13780-13789. CrossRef Medline

Hipp JF, Hawellek DJ, Corbetta M, Siegel M, Engel AK (2012) Large-scale cortical correlation structure of spontaneous oscillatory activity. Nat Neurosci 15:884-890. CrossRef Medline

Hua K, Zhang J, Wakana S, Jiang H, Li X, Reich DS, Calabresi PA, Pekar JJ, van Zijl PC, Mori S (2008) Tract probability maps in stereotaxic spaces:
Analyses of white matter anatomy and tract-specific quantification. Neuroimage 39:336-347. CrossRef Medline

Khan U A, Liu L, Provenzano FA, Berman DE, Profaci CP, Sloan R, Mayeux R, Duff KE, Small SA (2014) Molecular drivers and cortical spread of lateral entorhinal cortex dysfunction in preclinical Alzheimer's disease. Nat Neurosci 17:304-311. Medline

Kim T, Vidal GS, Djurisic M, William CM, Birnbaum ME, Garcia KC, Hyman BT, Shatz CJ (2013) Human LilrB2 is a $\beta$-amyloid receptor and its murine homolog PirB regulates synaptic plasticity in an Alzheimer's model. Science 341:1399-1404. CrossRef Medline

Klupp E, Förster S, Grimmer T, Tahmasian M, Yakushev I, Sorg C, Yousefi BH, Drzezga A (2014) In Alzheimer's disease, hypometabolism in lowamyloid brain regions may be a functional consequence of pathologies in connected brain regions. Brain Connect 4:371-383. CrossRef Medline

Lachaux JP, Rodriguez E, Martinerie J, Varela FJ (1999) Measuring phase synchrony in brain signals. Hum Brain Mapp 8:194-208. CrossRef Medline

Li R, Wu X, Chen K, Fleisher AS, Reiman EM, Yao L (2013) Alterations of directional connectivity among resting-state networks in Alzheimer disease. AJNR Am J Neuroradiol 34:340 -345. CrossRef Medline

López ME, Bruña R, Aurtenetxe S, Pineda-Pardo JÁ, Marcos A, Arrazola J, Reinoso AI, Montejo P, Bajo R, Maestú F (2014) Alpha-band hypersynchronization in progressive mild cognitive impairment: a magnetoencephalography study. J Neurosci 34:14551-14559. CrossRef Medline

Mattsson N, Andreasson U, Persson S, Carrillo MC, Collins S, Chalbot S, Cutler N, Dufour-Rainfray D, Fagan AM, Heegaard NH, Robin Hsiung GY, Hyman B, Iqbal K, Käser SA, Lachno DR, Lleó A, Lewczuk P, Molinuevo JL, Parchi P, Regeniter A, Rissman RA, Rosenmann H, Sancesario G, Schröder J, Shaw LM, Teunissen CE, Trojanowski JQ, Vanderstichele H, Vandijck M, Verbeek MM, Zetterberg H, Blennow K; Alzheimer's Association QC Program Work Group (2013) CSF biomarker variability in the Alzheimer's Association quality control program. Alzheimers Dement 9:251-261. CrossRef Medline

Pereda E, Quiroga RQ, Bhattacharya J (2005) Nonlinear multivariate analysis of neurophysiological signals. Prog Neurobiol 77:1-37. CrossRef Medline

Pineda-Pardo JA, Garcés P, López ME, Aurtenetxe S, Cuesta P, Marcos A, Montejo P, Yus M, Hernández-Tamames JA, del Pozo F, Becker JT, Maestú F (2014) White matter damage disorganizes brain functional networks in amnestic mild cognitive impairment. Brain Connect 4:312-322. CrossRef Medline

Serrano-Pozo A, Frosch MP, Masliah E, Hyman BT (2011) Neuropathological alterations in Alzheimer disease. Cold Spring Harb Perspect Med 1.

Sheline YI, Raichle ME, Snyder AZ, Morris JC, Head D, Wang S, Mintun MA (2010) Amyloid plaques disrupt resting state default mode network connectivity in cognitively normal elderly. Biol Psychiatry 67:584-587. CrossRef Medline

Spires-Jones TL, Hyman BT (2014) The intersection of amyloid beta and tau at synapses in Alzheimer's disease. Neuron 82:756-771. CrossRef Medline

Stam CJ (2010) Use of magnetoencephalography (MEG) to study functional brain networks in neurodegenerative disorders. J Neurol Sci 289: 128-134. CrossRef Medline

Tadel F, Baillet S, Mosher JC, Pantazis D, Leahy RM (2011) Brainstorm: a user-friendly application for MEG/EEG analysis. Comput Intell Neurosci 2011:879716. Medline

van Rossum IA, Visser PJ, Knol DL, van der Flier WM, Teunissen CE, Barkhof F, Blankenstein M a, Scheltens P (2012) Injury markers but not amyloid markers are associated with rapid progression from mild cognitive impairment to dementia in Alzheimer's disease. J Alzheimers Dis 29:319327. Medline

Vlassenko AG, Benzinger TL, Morris JC (2012) PET amyloid-beta imaging in preclinical Alzheimer's disease. Biochim Biophys Acta 1822:370-379. CrossRef Medline

Wang L, Brier MR, Snyder AZ, Thomas JB, Fagan AM, Xiong C, Benzinger TL, Holtzman DM, Morris JC, Ances BM (2013) Cerebrospinal fluid A $\beta 42$, phosphorylated Tau181, and resting-state functional connectivity. JAMA Neurol 70:1242-1248. Medline 